\title{
RELATIONS BETWEEN GEOMETRIC CONVEXITY, DOUBLING MEASURES AND PROPERTY $\Gamma$
}

\author{
LUIS A. CAFFARELLI AND MICHAEL G. CRANDALL
}

(Communicated by Thomas Schlumprecht)

\begin{abstract}
In this article it is shown that the three conditions on the norm $\|\cdot\|$ of a Banach space called "geometric convexity", "balanced" and "doubling" in an earlier work by the authors related to eikonal equations are in fact all equivalent. Moreover, each of them is equivalent to a condition called "Property $\Gamma$ " by Ganichev and Kalton. A fifth condition, that the second derivative of the function $t \mapsto\|x+t y\|$ is a doubling measure on [-2,2] for suitable $x, y \in X$, is also equivalent to the various other properties, and this formulation occupies a central place in the analysis.
\end{abstract}

\section{INTRODUCTION}

Let $X$ be a real Banach space when equipped with the continuously Fréchet differentiable norm $\|\cdot\|$. Let $N(x)=D\|x\|$ be the gradient of $\|\cdot\|$ at $x \in X \backslash\{0\}$ so that $N: X \backslash\{0\} \rightarrow X^{*}$, the dual of $X$. Denote the value of $x^{*} \in X^{*}$ at $x \in X$ by $\left\langle x, x^{*}\right\rangle$. Regarding $X$ as fixed throughout, we say that $\|\cdot\|$ is geometrically convex if there are numbers $0<r$ and $2<\Lambda$ such that

$$
\|x+2 y\|-\|x\|-2\langle y, N(x)\rangle \geq \Lambda(\|x+y\|-\|x\|-\langle y, N(x)\rangle),
$$

for $x, y \in X,\|y\| \leq r\|x\|, x \neq 0$.

This condition was introduced in the finite dimensional case in 2, wherein results were proved that were used in [1] to characterize all the solutions of certain Hamilton-Jacobi equations on $\mathcal{R}^{n} \backslash \mathcal{S}$ where $\mathcal{S}$ has vanishing Hausdorff 1 measure. We will not refer further to differential equations in this paper beyond this mention of the original purpose of introducing condition (1.1).

One result of this note is that the following condition is equivalent to geometric convexity: there exists $r_{1}>0$ such that all of the measures

$$
\frac{d^{2}}{d t^{2}}\|x+t y\|, \quad\|y\| \leq r_{1}\|x\|,
$$

are doubling on $-2 \leq t \leq 2$ with a common doubling constant (see Section 2 below). Note that the convexity of $t \rightarrow\|x+t y\|$ guarantees that the leftmost expression in (1.2) is sensible as a nonnegative Borel measure on $\mathcal{R}$.

Received by the editors September 9, 2011 and, in revised form, June 25, 2012 and July 11, 2012 .

2010 Mathematics Subject Classification. Primary 46B20.

Key words and phrases. Banach space, Property $\Gamma$, geometric convexity, doubling measures.

The first author was supported in part by NSF Grant DMS-1160802. 
We remark that the inelegant " $x \neq 0$ " in (1.1) and elsewhere below is due to $\|\cdot\|$ not being differentiable at the origin, and it could be replaced by " $\|x\|=1$ ", as $N(x)$ is positive homogeneous of degree 0 . This issue is not a concern in (1.2), as $x=0$ is a trivial case.

Introducing the function $h: X \backslash\{0\} \times X \rightarrow \mathcal{R}$ given by

$$
h(x, z)=\|z\|-\|x\|-\langle z-x, N(x)\rangle=\|z\|-\langle z, N(x)\rangle,
$$

which is the error between $\|z\|$ and the first order Taylor expansion of $\|z\|$ about $x$, (1.1) may be written

$$
h(x, x+2 y) \geq \Lambda h(x, x+y) \text { for }\|y\| \leq r\|x\|, x \neq 0 .
$$

Two additional properties employed in 2 are, first, that there are constants $r, K$ such that

$$
h(x, x-y) \leq K h(x, x+y) \quad \text { for } \quad\|y\| \leq r\|x\|, x \neq 0,
$$

which is called "balanced" in [2], and, second, that there are constants $r, T$ such that

$$
h(x, x+2 y) \leq T h(x, x+y) \quad \text { for } \quad\|y\| \leq r\|x\|, x \neq 0,
$$

which is called "doubling" in [2. We will take care so that no confusion results from the two definitions of "doubling" in this note.

In Section 2 we collect some simple facts which are used in Section 3 to prove the equivalence of the doubling condition on the measures (1.2) and geometric convexity. In fact, it turns out that all three conditions, geometric convexity, balanced in the sense of (1.5) and doubling in the sense of (1.6) are equivalent, as they are each equivalent to the doubling conditions on the measures. In a main result of [2] it was assumed that the norm was geometrically convex and balanced; it is now seen that "balanced" need not have been assumed separately, as it is implied by geometric convexity. The doubling condition (1.6) was used in a different manner in [2] and did not appear as an assumption in the main result.

Another of the main results of this note is that a condition called "Property $\Gamma$ " by Ganichev and Kalton in [3] and [4] is also equivalent to the each of the four conditions already discussed. This significantly extends the class of spaces for which geometric convexity was known to hold, as Ganichev and Kalton present a interesting array of spaces with Property $\Gamma$. It also provides alternative characterizations of Property $\Gamma$. Property $\Gamma$ is recalled in Section 4 where the equivalence is established. Results in [4] are also suggestive of the equivalences mentioned in the preceding paragraph.

\section{Preliminaries}

There are many equivalent ways to define the notion of a doubling measure on an interval. Here, we say that a nonnegative finite Borel measure $\mu$ on an interval $I$ is doubling if there exists a constant $C \geq 1$ such that if $I_{1}, I_{2}$ are adjacent subintervals of $I$ with $\left|I_{1}\right|=\left|I_{2}\right|$, then

$$
\mu\left(I_{1}\right) \leq C \mu\left(I_{2}\right)
$$

where $\left|I_{1}\right|$ is the length of $I_{1}$, etc., in which case we say that $\mu$ is doubling with constant $C$. Doubling measures do not have point masses, so it doesn't matter if the intervals are open or closed, etc., and we write $\mu(a, b)$ for any of $\mu((a, b)), \mu([a, b])$, etc. 
For our purposes, we put $I=[-2,2]$ and first recall another condition equivalent to (2.1) when $I_{1}$ is required to be to the left of $I_{2}$.

Lemma 2.1. Let $\mu$ be a finite Borel measure on $[-2,2]$ with the property that there are constants $0<\gamma<1,0<C_{0}$ for which

$$
\mu(a, a+\gamma(b-a)) \leq C_{0} \mu(a+\gamma(b-a), b)
$$

whenever $-2 \leq a<b \leq 2$. Then

$$
\mu\left(a, \frac{a+b}{2}\right) \leq C \mu\left(\frac{a+b}{2}, b\right) \quad \text { for } \quad-2 \leq a<b \leq 2,
$$

where

$$
C:=C_{0}\left(\sum_{i=0}^{N-1}\left(1+C_{0}\right)^{N-(i+1)}\right),
$$

and $N$ is the least integer satisfying $N \geq-\ln (2) / \ln (1-\gamma)$.

Proof. In order to interpret (2.2), notice that it is equivalent to the condition that $\mu\left(I_{1}\right) \leq C_{0} \mu\left(I_{2}\right)$ provided that $I_{1}, I_{2}$ are adjacent subintervals of $[-2,2], I_{1}$ is to the left of $I_{2}$ and $\left|I_{1}\right|=(\gamma /(1-\gamma))\left|I_{2}\right|$. The conclusion (2.3) shows that the condition (2.1) is satisfied if $I_{1}$ is to the left of $I_{2}$ and $\left|I_{1}\right|=\left|I_{2}\right|$. In use, we will deal with intervals "to the other side" by replacing $\mu$ by $\tilde{\mu}(a, b):=\mu(-b,-a)$.

Fix $-2 \leq a<b \leq 2$ and define

$$
a_{0}=a, a_{n+1}=a_{n}+\gamma\left(b-a_{n}\right)=\alpha a_{n}+(1-\alpha) b, n=1,2, \ldots,
$$

where $\alpha=1-\gamma$. Then

$$
a_{n}=\alpha^{n} a+\left(1-\alpha^{n}\right) b
$$

is a convex combination of $a$ and $b$, while (2.2) with $a$ replaced by $a_{n}$ yields

$$
\mu\left(a_{n}, a_{n+1}\right) \leq C_{0} \mu\left(a_{n+1}, b\right),
$$

and we therefore also have

$$
\begin{aligned}
\mu\left(a_{n}, b\right) & =\mu\left(a_{n}, a_{n+1}\right)+\mu\left(a_{n+1}, b\right) \\
& \leq C_{0} \mu\left(a_{n+1}, b\right)+\mu\left(a_{n+1}, b\right)=\left(C_{0}+1\right) \mu\left(a_{n+1}, b\right) .
\end{aligned}
$$

Let $N$ be the least integer for which

$$
\alpha^{N}=(1-\gamma)^{N} \leq \frac{1}{2} \text { or } a_{N}=\alpha^{N} a+\left(1-\alpha^{N}\right) b \geq \frac{a+b}{2} .
$$

By (2.8),

$$
\mu\left(a_{n}, b\right) \leq\left(1+C_{0}\right)^{N-n} \mu\left(a_{N}, b\right) \text { for } n=0, \ldots, N,
$$

and then by this and (2.7),

$$
\begin{aligned}
\mu\left(a, a_{N}\right)=\sum_{i=0}^{N-1} \mu\left(a_{i}, a_{i+1}\right) & \leq C_{0} \sum_{i=0}^{N-1} \mu\left(a_{i+1}, b\right) \\
& \leq C_{0}\left(\sum_{i=0}^{N-1}\left(1+C_{0}\right)^{N-(i+1)}\right) \mu\left(a_{N}, b\right) .
\end{aligned}
$$

Since $a_{N} \geq(a+b) / 2$, we have proved (2.3). 
Suppose that $I$ is an interval and $\varphi: I \rightarrow \mathcal{R}$ is convex and continuously differentiable, and let

$$
\mu:=\frac{d^{2}}{d t^{2}} \varphi(t)
$$

If $s, t \in I$ and $s \leq t$, then $\mu(s, t)=\varphi^{\prime}(t)-\varphi^{\prime}(s)$ and

$$
\varphi(t)-\varphi(s)-(t-s) \varphi^{\prime}(s)=\int_{s}^{t}(t-\tau) d \mu(\tau) .
$$

We next collect some further simple results, unified by their one dimensional character, for later use. They may be skipped now and returned to when cited again in Section 3 Meantime, we remark that the assertion (2.11) of Lemma 2.2 below is used to establish (1.4) as a direct consequence of the assumed doubling character of the measure on the left of (1.2), if so assumed, while (1.5) follows similarly from (2.13) as does (1.6) from (2.12), all applied to $\varphi(t)=h(x, x+t y)$.

Lemma 2.2. Suppose $\varphi:[-2,2] \rightarrow \mathcal{R}$ is convex, nonnegative, continuously differentiable and $\varphi(0)=\varphi^{\prime}(0)=0$. Suppose also that $\mu:=\frac{d^{2}}{d t^{2}} \varphi(t)$ is doubling on $[-2,2]$ with constant $C$. Then there exist constants $0<T, K, 2<\Lambda$, depending only on $C$, such that

$$
\begin{aligned}
& \Lambda \varphi(t) \leq \varphi(2 t) \quad \text { for }-1 \leq t \leq 1 \\
& \varphi(2 t) \leq T \varphi(t), \quad \text { for }-1 \leq t \leq 1
\end{aligned}
$$

and

$$
\varphi(-t) \leq K \varphi(t) \text { for }-2 \leq t \leq 2 .
$$

In fact, we may choose $T=16 C^{2}, K=4 C^{2}, \Lambda=(8 C-1) /(4 C-1)$.

Proof. We assume that $\mu$ is doubling with constant $C \geq 1$ and begin with some simple observations. To establish the various claims, it suffices to assume that $0 \leq t$, for $\tilde{\varphi}(t):=\varphi(-t)$ satisfies the same conditions as $\varphi$. First,

$$
\begin{aligned}
C \mu(0, t / 2)= & \frac{C}{2} \mu(0, t / 2)+\frac{C}{2} \mu(0, t / 2) \geq \frac{1}{2}(C \mu(0, t / 2)+\mu(t / 2, t)) \\
& \geq \frac{1}{2}(\mu(0, t / 2)+\mu(t / 2, t))=\frac{1}{2} \mu(0, t) .
\end{aligned}
$$

Similarly,

$$
C \mu(t / 2, t) \geq \frac{1}{2} \mu(0, t) .
$$

It follows from (2.10), (2.14) that

$$
\begin{aligned}
C \varphi(t)=C \int_{0}^{t}(t-\tau) d \mu(\tau) & \geq C \frac{t}{2} \int_{0}^{t / 2} d \mu(\tau) \\
& =C \frac{t}{2} \mu(0, t / 2) \geq \frac{t}{4} \mu(0, t) .
\end{aligned}
$$

On the other hand,

$$
t \mu(0, t) \geq \int_{0}^{t}(t-\tau) d \mu(\tau)=\varphi(t) .
$$

Combining (2.16) and (2.17), we have

$$
\frac{t}{4 C} \mu(0, t) \leq \varphi(t) \leq t \mu(0, t) .
$$


To establish (2.11), we seek $\Lambda>2$ such that

or

$$
\varphi(2 t)=\int_{0}^{2 t}(2 t-\tau) d \mu(\tau) \geq \Lambda \int_{0}^{t}(t-\tau) d \mu(\tau)=\Lambda \varphi(t)
$$

$$
\begin{gathered}
(\Lambda-1) \int_{0}^{t} \tau d \mu(\tau)+\int_{t}^{2 t}(2 t-\tau) d \mu(\tau) \geq(\Lambda-2) t \int_{0}^{t} d \mu(\tau) \\
=(\Lambda-2) t(\mu(0, t / 2)+\mu(t / 2, t)) .
\end{gathered}
$$

Using

$$
\int_{0}^{t} \tau d \mu(\tau) \geq \frac{t}{2} \int_{t / 2}^{t} d \mu(\tau)=\frac{t}{2} \mu(t / 2, t) \geq \frac{t}{2 C} \mu(0, t / 2),
$$

we see that

$$
\begin{aligned}
(\Lambda-1) \int_{0}^{t} \tau d \mu(\tau) & =\frac{\Lambda-1}{2} \int_{0}^{t} \tau d \mu(\tau)+\frac{\Lambda-1}{2} \int_{0}^{t} \tau d \mu(\tau) \\
& \geq \frac{\Lambda-1}{2} \frac{t}{2 C} \mu(0, t / 2)+\frac{\Lambda-1}{2} \frac{t}{2} \mu(t / 2, t) .
\end{aligned}
$$

Thus (2.19) holds provided that

$$
(\Lambda-1) \geq 4 C(\Lambda-2) \text { and } \Lambda-1 \geq 4(\Lambda-2),
$$

with the second inequality a consequence of the first, and we may choose $\Lambda>2$ accordingly. In fact, it suffices to take

$$
\Lambda=\frac{8 C-1}{4 C-1}
$$

Next we establish (2.12). Observe that by (2.14) and (2.18),

$$
\varphi(2 t) \leq 2 t \mu(0,2 t) \leq 4 t C \mu(0, t) \leq 16 C^{2} \varphi(t) .
$$

We conclude that (2.12) holds with $T=16 C^{2}$.

We conclude by establishing (2.13). Applying (2.18) to $\tilde{\varphi}(t):=\varphi(-t)$, followed by the doubling assumption and another application of (2.18), we find

$$
\varphi(-t) \leq t \mu(-t, 0) \leq t C \mu(0, t) \leq 4 C^{2} \varphi(t) .
$$

Remark 2.3. Lemma 2.2 is a variant of results in [4]; see Lemma 2.1 and Proposition 2.10 of 4 in conjunction with Section 4 below.

Any smooth convex $\varphi$ satisfying $2 t^{4} \leq \varphi(t) \leq 8 t^{4}$ satisfies (2.11), (2.12), and (2.13) with $\Lambda=4, T=64, K=2$, but $\varphi^{\prime \prime}$ may vanish on intervals, which cannot happen for nontrivial doubling measures. However, the next lemma shows that a vestige of doubling remains in each case.

Lemma 2.4. Let $\varphi:[-2,2]: \rightarrow \mathcal{R}$ be $C^{1}$, convex, $\varphi(0)=\varphi^{\prime}(0)=0$ and $\mu:=$ $d^{2} \varphi(t) / d t^{2}$.

(a) Suppose that $\Lambda>2$ and $\varphi(2) \geq \Lambda \varphi(1)$. Then

$$
\mu(0, \beta) \leq \frac{4}{\Lambda-2} \mu(\beta, 2), \quad \text { where } \beta=\frac{\Lambda-2}{2(\Lambda-1)} .
$$


(b) Suppose that $\varphi(-2) \leq K \varphi(2)$. Then

$$
\mu(-1,0) \leq 2 K \mu(0,2) .
$$

(c) Suppose that $\varphi(2) \leq T \varphi(1)$. Then

$$
\mu(1,3 / 2) \leq 2 T \mu(0,1) .
$$

Proof. We establish (a). By assumption, for $0<\beta \leq 1$,

$$
\begin{aligned}
& \varphi(2)=\int_{0}^{2}(2-\tau) d \mu(\tau)=\int_{\beta}^{2}(2-\tau) d \mu(\tau)+\int_{0}^{\beta}(2-\tau) d \mu(\tau) \\
& \geq \Lambda \varphi(1)=\Lambda \int_{0}^{1}(1-\tau) d \mu(\tau) \geq \Lambda \int_{0}^{\beta}(1-\tau) d \mu(\tau) .
\end{aligned}
$$

Rearranging the inequality between the third expression from the left and the quantity on the far right hand side, and making some simple estimates, one finds

$$
\begin{aligned}
(2-\beta) \mu(\beta, 2) & \geq \int_{\beta}^{2}(2-\tau) d \mu(\tau) \\
& \geq \int_{0}^{\beta}(\Lambda-2-(\Lambda-1) \tau) d \mu(\tau) \geq(\Lambda-2-(\Lambda-1) \beta) \mu(0, \beta) .
\end{aligned}
$$

Plug in the $\beta$ of (2.21) above and use $(\Lambda-2)(2-\beta) \leq 4(\Lambda-2-(\Lambda-1) \beta)$ to find the estimate of (2.21). This last step was used only to have a cleaner looking constant in (2.21).

To prove (b), we note that

$$
\mu(-1,0) \leq \int_{-2}^{0}(2+\tau) d \mu(\tau)=\varphi(-2) \leq K \varphi(2) \leq 2 K \mu(0,2),
$$

while (c) follows from

$$
\frac{1}{2} \mu(1,3 / 2) \leq \int_{0}^{2}(2-\tau) d \mu(\tau)=\varphi(2) \leq T \varphi(1) \leq T \mu(0,1) .
$$

\section{Geometric Convexity And Doubling measures}

Recall the notation (1.3).

Theorem 3.1. The following conditions are equivalent:

(i) There exist $r>0, \Lambda>2$ such that

$$
h(x, x+2 y) \geq \Lambda h(x, x+y) \quad \text { for } \quad\|y\| \leq r\|x\|, x \neq 0 .
$$

(ii) There exist $r, K>0$ such that

$$
h(x, x-y) \leq K h(x, x+y) \quad \text { for } \quad\|y\| \leq r\|x\|, x \neq 0 .
$$

(iii) There exist $r, T>0$ such that

$$
h(x, x+2 y) \leq T h(x, x+y) \quad \text { for } \quad\|y\| \leq r\|x\|, x \neq 0 .
$$

(iv) There exists $r_{1}>0$ such that all of the measures

$$
\mu_{x, y}:=\frac{d^{2}}{d t^{2}}\|x+t y\|, \quad\|y\| \leq r_{1}\|x\|,
$$

have a common doubling constant on $|t| \leq 2$. 
Proof. In order to show that (i)-(iii) imply (iv), we will use the estimates of Lemma 2.4 and parlay them into estimates of the form (2.2) via changes of variables. Then Lemma 2.1 provides the required doubling constant. Let $x \neq 0,\|y\| \leq r\|x\|$ and put

$$
\varphi(t):=h(x, x+t y)=\|x+t y\|-\|x\|-t\langle y, N(x)\rangle
$$

and note that $\varphi$ is convex, nonnegative, $\varphi(0)=\varphi^{\prime}(0)=0$, and $\frac{d^{2}}{d t^{2}} \varphi(t)=\mu_{x, y}$. We need to avoid $x+t y=0$, as $\varphi$ is not differentiable at such values of $t$, and we can guarantee that this does not occur for $|t| \leq 2$ by assuming that $\|y\| \leq r\|x\|$ and $r<1 / 2$, as we now impose.

The changes of variables we will use are of the form

$$
\tilde{\mu}:=\frac{d^{2}}{d t^{2}}\|x+A y+t B y\|,
$$

where $A \in \mathcal{R}, B \in \mathcal{R} \backslash\{0\}$. We will impose that

$$
(|B|+r|A|)\|y\| \leq r\|x\|
$$

which guarantees that $\|B y\| \leq r\|x+A y\|$. Observe that for $c<d$,

$$
\tilde{\mu}(c, d)= \begin{cases}B \mu_{x, y}(A+c B, A+d B) & \text { if } \quad B>0, \\ |B| \mu_{x, y}(A+d B, A+c B) & \text { if } \quad B<0 .\end{cases}
$$

Let us prove that (i) implies (iv). With $\varphi$ given by (3.1), (i) implies $\varphi(2) \geq \Lambda \varphi(1)$, and (2.21) of Lemma 2.4 (a) then applies to yield

$$
\mu_{x, y}(0, \beta) \leq \frac{4}{\Lambda-2} \mu_{x, y}(\beta, 2)
$$

for the $\beta$ of (2.21). Let $-2 \leq a<b \leq 2$. The estimate above also holds for $\tilde{\mu}$ of (3.2), in which we choose $A=a, B=(b-a) / 2$. Then $\tilde{\mu}(0, \beta) \leq(4 /(\Lambda-2)) \tilde{\mu}(\beta, 2)$ becomes, via (3.4),

$$
\mu_{x, y}\left(a, a+\frac{\beta}{2}(b-a)\right) \leq \frac{4}{\Lambda-2} \mu_{x, y}\left(a+\frac{\beta}{2}(b-a), b\right) .
$$

We have established (2.2) with $\gamma=\beta / 2$, so (2.3) holds for $\mu_{x, y}$ where $C$ depends only on $\Lambda$. This shows that if $I_{1}, I_{2}$ are adjacent subintervals of $[-2,2]$ of equal length and $I_{1}$ is to the left of $I_{2}$, then $\mu_{x, y}\left(I_{1}\right) \leq C \mu_{x, y}\left(I_{2}\right)$. To handle the situation where $I_{2}$ is to the left of $I_{1}$, replace $y$ by $-y$ and notice that if

$$
\tilde{\mu}=\frac{d^{2}}{d t^{2}}\|x-t y\|
$$

then $\tilde{\mu}(a, b)=\mu(-b,-a)$.

The restriction (3.3) is satisfied here if $\|y\| \leq r /(2(1+r))\|x\|$, as $|A|,|B| \leq 2$, and we may take $r_{1}=r /(2(1+r))$ in (ii).

To establish that (iv) implies (i), we simply apply (2.11) of Lemma 2.2 with $t=1$ to $\varphi$ of (3.1). The result is (i) with $r=r_{1}$. 
The equivalence of (ii) and (iv) and (iii) and (iv) are established in an entirely similar manner. For example, to show that (ii) implies (iv), one uses $\tilde{\mu}(-1,0) \leq$ $2 K \tilde{\mu}(0,2)$ with $A=(2 a+b) / 3, B=(b-a) / 3$ and finds, via (3.4),

$$
\mu_{x, y}\left(a, a+\frac{1}{3}(b-a)\right) \leq 2 K \mu_{x, y}\left(a+\frac{1}{3}(b-a), b\right)
$$

and so forth. The pairwise equivalence of (i), (ii), and (iii) follows from the fact that they are each equivalent to (iv).

\section{Property $\Gamma$ of Ganichev and Kalton}

In [3] and [4], $\|\cdot\|$ is said to have Property $\Gamma$ if

$$
\begin{aligned}
& \exists \gamma \in(0,1] \text { such that if }\langle y, N(x)\rangle=0, \\
& \text { then }\|x+y\| \geq\|x\|+\gamma\langle y, N(x+y)\rangle .
\end{aligned}
$$

Theorem 4.3 of 4 states that $\|\cdot\|$ has Property $\Gamma$ iff there is an $r>1$ and a constant $\gamma \geq 2$ such that $f(x):=\|x\|^{r}$ satisfies

$$
f(x+2 y)+f(x-2 y)-2 f(x) \leq \gamma(f(x+y)+f(x-y)-2 f(x))
$$

for $x, y \in X$. Moreover, if Property $\Gamma$ holds, then for every $r>1$ there is a $\gamma$ such that (4.2) holds.

A general continuous convex function $f: X \rightarrow \mathcal{R}$ is said in 4 to be tame with constant $\gamma$ if it satisfies (4.2). Note that if $f$ is a tame convex function with constant $\gamma$, then $\varphi: \mathcal{R} \rightarrow \mathcal{R}$ defined by

$$
\varphi(t):=f(x+t y)
$$

is tame with constant $\gamma$ for any $x, y \in X$.

Theorem 4.1. Each of conditions (i)-(iv) of Theorem 3.1 is equivalent to the condition that $\|\cdot\|$ has Property $\Gamma$.

Proof. We begin by showing that if $\|\cdot\|$ has Property $\Gamma$, then (iii) of Theorem 3.1 holds (and hence so do (i), (ii), (iv)). It suffices to show that if $f(x):=\|x\|^{2}$ is tame, then $\|\cdot\|$ is doubling in the sense of Theorem 3.1 (iii). We assume that $x \neq 0$ below. If

$$
\langle y, N(x)\rangle=0,
$$

the defining inequality of "doubling" (1.6),

$$
h(x, x+2 y) \leq T h(x, x+y),
$$

amounts to

$$
\|x+2 y\|-\|x\| \leq T(\|x+y\|-\|x\|) .
$$

The function $\varphi(t):=\|x+t y\|^{2}-\|x\|^{2}$ is convex, differentiable, and, using (4.3),

$$
\varphi(0)=0, \varphi^{\prime}(0)=2\|x\|\langle y, N(x)\rangle=0 .
$$


Thus $\varphi$ is nonnegative. Since $\varphi$ is also tame with some constant $\gamma$, it follows from Lemma 2.1 of [4] that

$$
\|x+2 y\|^{2}-\|x\|^{2}=\varphi(2) \leq B \varphi(1)=B\left(\|x+y\|^{2}-\|x\|^{2}\right),
$$

where $B=\gamma^{3}$, or

$$
\|x+2 y\|-\|x\| \leq\left(\frac{\|x+y\|+\|x\|}{\|x+2 y\|+\|x\|} B\right)(\|x+y\|-\|x\|) .
$$

We may assume that $\|x\|=1$. If $\|y\| \leq 1$, then

$$
\frac{\|x+y\|+\|x\|}{\|x+2 y\|+\|x\|}=\frac{\|x+y\|+1}{\|x+2 y\|+1} \leq \frac{3}{2},
$$

where we used $\|x+2 y\| \geq\|x\|=1$ (see (4.17) below). If $\|y\| \geq 1$, then

$$
\frac{\|x+y\|+1}{\|x+2 y\|+1} \leq \frac{2+\|y\|}{2\|y\|} \leq \frac{3}{2}
$$

Thus, if $\langle y, N(x)\rangle=0$, we have

$$
h(x, x+2 y) \leq T h(x, x+y),
$$

where $T=3 B / 2$, or doubling in the tangent plane with no restriction of the form $\|y\| \leq r\|x\|$. Here "in the tangent plane" corresponds to the assumption $\langle y, N(x)\rangle=0$. The proof of Theorem 6.5 of [2] now implies the full doubling condition (iii) of Theorem 3.1. For the reader's convenience, at the suggestion of the referee, we present this proof.

We assume that $\|x\|=1$ and produce $r, T>0$ such that if $\|y\| \leq r\|x\|$, then (4.6) holds. Given $y$, we write

$$
y=\alpha x+\varepsilon x^{\perp}, \quad \text { where } \alpha=\langle y, N(x)\rangle \text { and } \varepsilon=\|y-\alpha x\| .
$$

The vector $x^{\perp}$ is defined by (4.7) if $\varepsilon \neq 0$ and further satisfies $\left\|x^{\perp}\right\|=1$ (by the choice of $\varepsilon$ ) and $\left\langle x^{\perp}, N(x)\right\rangle=0$ (by the choice of $\alpha$ ). If $\varepsilon=0$, we choose $x^{\perp}$ to be any unit vector satisfying $\left\langle x^{\perp}, N(x)\right\rangle=0$. We will assume for the moment that

$$
-\frac{1}{2}<\alpha
$$

and strengthen this later. Note that if $\varepsilon=0$ and (4.8) holds, then $h(x, x+2 y)=$ $h(x, x+y)=0$, and (4.6) holds with any $T$ whatsoever. Thus we may assume that $\varepsilon>0$. Using (4.6) (with $(1+2 \alpha) x$ in place of $x$ and $\varepsilon x^{\perp}$ in place of $y$ ), we have

$$
\begin{aligned}
h(x, x+2 y) & =h\left(x, x+2\left(\alpha x+\varepsilon x^{\perp}\right)\right) \\
& =h\left((1+2 \alpha) x,(1+2 \alpha) x+2 \varepsilon x^{\perp}\right) \\
& =\left\|(1+2 \alpha) x+2 \varepsilon x^{\perp}\right\|-(1+2 \alpha)\|x\| \\
& \leq T\left(\left\|(1+2 \alpha) x+\varepsilon x^{\perp}\right\|-(1+2 \alpha)\|x\|\right) \\
& =T(1+2 \alpha)\left(\left\|x+\frac{\varepsilon}{1+2 \alpha} x^{\perp}\right\|-\|x\|\right) .
\end{aligned}
$$

In the same way,

$$
\begin{aligned}
h(x, x+y) & =\left\|(1+\alpha) x+\varepsilon x^{\perp}\right\|-(1+\alpha)\|x\| \\
& =(1+\alpha)\left(\left\|x+\frac{\varepsilon}{1+\alpha} x^{\perp}\right\|-\|x\|\right),
\end{aligned}
$$


which in combination with (4.9) yields

$$
h(x, x+2 y) \leq T \frac{1+2 \alpha}{1+\alpha} \frac{\left\|x+\frac{\varepsilon}{1+2 \alpha} x^{\perp}\right\|-\|x\|}{\left\|x+\frac{\varepsilon}{1+\alpha} x^{\perp}\right\|-\|x\|} h(x, x+y) .
$$

Now define

$$
f(t):=\left\|x+t \varepsilon x^{\perp}\right\|-\|x\| .
$$

The function $f$ is convex and $f(0)=f^{\prime}(0)=0$. Moreover, by (4.6),

$$
f(2 t) \leq T f(t)
$$

which implies that if $f(t)=0$ for any $t>0$, then $f(t)=0$ for all $t>0$, which is certainly not the case since $\varepsilon>0$. This is also the reason that (4.10) does not involve a division by 0 . Hence $f(1)>0$. Let

$$
g(t):=\frac{f(t)}{f(1)}
$$

The properties of $f$ above persist for $g$, and, in addition, $g(1)=1$; in particular,

$$
g(4) \leq T g(2) \leq T^{2} g(1)=T^{2} .
$$

Now the left hand side of

$$
\frac{g(4)-g(2)}{2} \leq \frac{g(4)-g(1)}{2} \leq \frac{T^{2}-1}{2}
$$

is a Lipschitz constant for $g$ on $[0,2]$. In terms of $g$, 4.10 states that

$$
h(x, x+2 y) \leq T \frac{1+2 \alpha}{1+\alpha} \frac{g\left(\frac{1}{1+2 \alpha}\right)}{g\left(\frac{1}{1+\alpha}\right)} h(x, x+y) .
$$

If $-\frac{1}{4}<\alpha$, then the arguments of $g$ above lie in [0,2]. Using the Lipschitz constant of $g$ established above, denote it by $L:=\left(T^{2}-1\right) / 2$, we conclude that

$$
\frac{g\left(\frac{1}{1+2 \alpha}\right)}{g\left(\frac{1}{1+\alpha}\right)} \leq \frac{g(1)+L\left|\frac{1}{1+2 \alpha}-1\right|}{g(1)-L\left|\frac{1}{1+\alpha}-1\right|}=\frac{1+L\left|\frac{1}{1+2 \alpha}-1\right|}{1-L\left|\frac{1}{1+\alpha}-1\right|} .
$$

It is now evident that there is a $r>0$, depending on $L$, such that (4.15) implies

$$
h(x, x+2 y) \leq 2 T h(x, x+y) \text { provided that }|\alpha| \leq r .
$$

Since $\|y\|=\left\|\alpha x+\varepsilon x^{\perp}\right\| \geq\|\alpha x\|=|\alpha|$, we have established (iii) of Theorem 3.1 with the $r$ above and the $2 T$ of this proof playing the role of the $T$ of (iii).

In the other direction, we show that condition (iv) of Theorem 3.1 that is, there is an $r>0$ such that all of the measures

$$
\frac{d^{2}}{d t^{2}}\|x+t y\|, \quad\|y\| \leq r\|x\|,
$$

have a common doubling constant on $[-2,2]$-implies (4.1).

Let $\langle y, N(x)\rangle=0$. Two elementary consequences of this that we will use are

$$
\left\{\begin{array}{l}
\text { (i) }\|x\| \leq\|x+a y\| \quad \text { for } \quad a \geq 0 \\
\text { (ii) }\langle y, N(x+a y)\rangle \geq 0 \text { for } \quad a \geq 0 .
\end{array}\right.
$$


Both follow from the convexity of $t \mapsto \varphi(t):=\|x+t y\|$ and $\varphi^{\prime}(0)=\langle y, N(x)\rangle=0$, so $\varphi(a) \geq \varphi(0)$ and $\varphi^{\prime}(a) \geq 0$ for $a \geq 0$.

To establish (4.1), we first note that if $\|y\| \geq 3\|x\|$, then

$$
\|x+y\|-\|x\| \geq\|y\|-2\|x\| \geq\left(1-\frac{2}{3}\right)\|y\| \geq \frac{1}{3}\langle y, N(x+y)\rangle,
$$

and (4.1) holds with $\gamma=1 / 3$. Thus we may assume that $\|y\|<3\|x\|$.

If $\|y\|<3\|x\|$, choose the least integer $n \geq 1$ such that

$$
\|y\| \leq 2 n r\|x\| \text {. }
$$

For $a \geq 0$, define

$$
\varphi(t):=\left\|x+a y+t\left(\frac{y}{2 n}\right)\right\|, \mu:=\frac{d^{2}}{d t^{2}} \varphi(t) .
$$

In view of (4.17) (i) and (4.18), $\|y\| / 2 n \leq r\|x+a y\|$, and then (4.16) implies that $\mu$ has a doubling constant $C \geq 1$ uniform in $a \geq 0$. Hence

$$
\begin{array}{r}
\varphi(2)-\varphi(0)-2 \varphi^{\prime}(0)=\int_{0}^{2}(2-\tau) d \mu(\tau) \geq \int_{0}^{1} d \mu(\tau)=\mu(0,1) \\
\geq \frac{1}{2 C} \mu(0,2)=\frac{1}{2 C}\left(\varphi^{\prime}(2)-\varphi^{\prime}(0)\right)
\end{array}
$$

or, using (4.17) (ii) as well,

$$
\begin{aligned}
& \left\|x+a y+\frac{y}{n}\right\|-\|x+a y\| \\
& \geq\left\|x+a y+\frac{y}{n}\right\|-\|x+a y\|-\frac{1}{n}\langle y, N(x+a y)\rangle \\
& \quad \geq \frac{1}{4 n C}\left(\left\langle y, N\left(x+a y+\frac{y}{n}\right)\right\rangle-\langle y, N(x+a y)\rangle\right) .
\end{aligned}
$$

Now take (4.20) with $a=k / n, k=0,1, \ldots, n-1$, to find

$$
\begin{aligned}
\left\|x+(k+1) \frac{y}{n}\right\| & -\left\|x+k \frac{y}{n}\right\| \\
& \geq \frac{1}{4 n C}\left(\left\langle y, N\left(x+(k+1) \frac{y}{n}\right)\right\rangle-\left\langle y, N\left(x+k \frac{y}{n}\right)\right\rangle\right)
\end{aligned}
$$

for $k=0,1, \ldots, n-1$. Adding these inequalities and recalling $\langle y, N(x)\rangle=0$ yields

$$
\|x+y\|-\|x\| \geq \frac{1}{4 n C}\langle y, N(x+y)\rangle,
$$

which is (4.1) with $\gamma=1 /(4 n C)$.

Thus if $\|y\| \geq 3\|x\|$, we may take $\gamma=1 / 3$ in (4.1), while if $\|y\|<3\|x\|$, the least integer $n \geq 1$ for which (4.18) holds is at most $(2 r+3) /(2 r)$, and (4.1) holds with $\gamma=2 r /((2 r+3) 4 C)$.

Remark 4.2. One of the results of [2], obtained with some sweat, is that $\mathcal{R}^{n}$ with the $p$-norm is geometrically convex for $1<p<\infty$. In 4 it is proved that general $L^{p}$ spaces have Property $\Gamma$ (see also 3]), that subspaces of quotient spaces of spaces with Property $\Gamma$ have Property $\Gamma$, that duals of spaces with Property $\Gamma$ have Property $\Gamma$, and other examples and properties are presented as well. Hence the case of $\mathcal{R}^{n}$ with the $p$-norm is subsumed by Theorem 4.1. However, an ingredient of the proof given in 2 was the passage from conditions in the tangent plane to the general case, which we still rely on here in the proof of Theorem 4.1. 


\section{REFERENCES}

[1] Luis A. Caffarelli and Michael G. Crandall, Distance functions and almost global solutions of eikonal equations, Comm. Partial Differential Equations 35 (2010), no. 3, 391-414, DOI 10.1080/03605300903253927. MR2748630(2012e:35026)

[2] Luis A. Caffarelli and Michael G. Crandall, The problem of two sticks, Expo. Math. 30 (2012), no. 1, 69-95, DOI 10.1016/j.exmath.2011.09.001. MR2899657

[3] M. Ganichev and N. J. Kalton, Convergence of the weak dual greedy algorithm in $L_{p}$-spaces, J. Approx. Theory 124 (2003), no. 1, 89-95, DOI 10.1016/S0021-9045(03)00133-3. MR2010781 (2005d:41043)

[4] M. Ganichev and N. J. Kalton, Convergence of the dual greedy algorithm in Banach spaces, New York J. Math. 15 (2009), 73-95. MR2481308(2010a:46033)

Department of Mathematics, University of Texas at Austin, Austin, Texas 78712

E-mail address: caffarel@math.utexas.eduu

Department of Mathematics, University of California at Santa Barbara, Santa Barbara, California 93106

E-mail address: crandall@math.ucsb.edu 